\title{
Operation of Medium-size Reverse Osmosis Plants with Optimal Energy Consumption
}

\author{
L. Palacin*, C. de Prada**, \\ F. Tadeo**, J. Salazar** \\ *Centro de Tecnologia Azucarera, Edificio Alfonso VIII, \\ 47011 Valladolid, Spain (e-mail: palacin@cta.uva.es) \\ ** Dpto. Ingeniería de Sistemas y Automática, \\ Fac. Ciencias, Univ. Valladolid, 47005 Valladolid, Spain
}

\begin{abstract}
This paper deals with the optimal operational strategy of a reverse osmosis (RO) plant for remote sites. The electricity supply to these plants comes usually from renewable energies (wind and solar), when they are not temporarily available, they are complemented by a diesel generator and batteries. The water demand of small settlements in arid regions suffers strong variations along a day. As the higher demand of water usually occurs when solar energy is more available, the operational expenses can be reduced by considering the RO plant as an active load. A good control strategy, will implement a variable operation point, taking into account the predictions of water demand, the expected variation of the available energy sources and the scheduling of cleaning operations in the RO plant, in order to optimize the energy use. In this paper a hybrid predictive control is proposed to implement this task. Simulations of a specific plant show that an adequate operation reduces the diesel energy consumption, while satisfying the variable water demand.
\end{abstract}

Keywords: Reverse Osmosis, Desalination Plants, Hybrid Predictive Control, Dynamic Optimization, Fault Simulation, Renewable Energy

\section{INTRODUCTION}

Reverse Osmosis (RO) is known to be an effective technique in arid or semi arid regions to produce drinkable water from brackish wells and sea water, Fritzmann et al. (2007). This is because RO plants need less energy, investment cost, space and maintenance than other alternative desalination processes (Gambier et al. 2007a); hence, it is the preferred desalination technique worldwide, Baker (2004), Wilf (2007).

In the particular case of water supply of villages and small settlements, small to medium-size RO plants are successfully used. In this case, energy consumption is commonly fulfilled by renewable energy sources, such as solar or wind, while diesel generators are needed in order to keep it operating when no renewable sources are available, Tadeo et al. (2009). This distribution of wind solar energy is selected to provide energy taking into account the lack of solar radiation during the night, and to compensate the wide variations in energy from wind generators. Moreover, batteries are used to store the temporal extra production of renewable energy during high availability of wind or solar radiation. At the same time, the daily variation of the water demand is well known, Porter (1990), with significant changes in small villages. Fig. 1 shows a typical water demand curve.

Typically, RO plants are designed to operate at a constant operation point, producing a constant flow of drinkable water. The variations of the consumption are compensated by big storage tanks at the end of the production line, which are costly and create losses by evaporation. In Palacin et al. (2009a) it was shown a better operation strategy, where water production varies along the day, adjusting water production and water consumption. This reduced significantly the storages size and decreased the lost of drinkable water by evaporation, the consumption of chemicals and the equipment cost. In Palacin et al. (2009b) an integrated design of a RO plant with a significant decrease in capital expenditure was presented.

(\% of the average flow) water demand

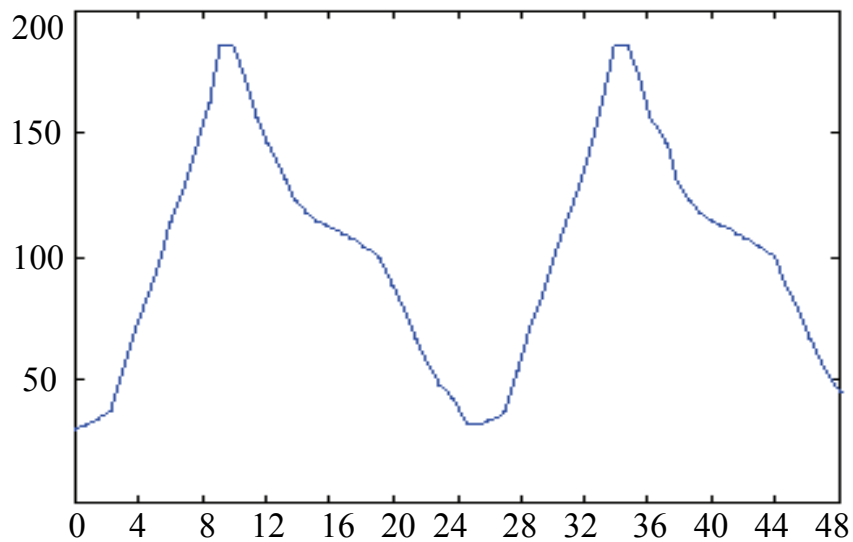

Fig. 1: Typical water demand curve during 48 hours.

Because the higher water consumption occurs when more renewable energy is available, the renewable energy consumption can be optimized and the auxiliary energy 
consumption (diesel) minimized, using a variable production policy, Salazar et al. (2010). The viability of a strategy that takes into account the expected renewable energy variations and the water demand curve was shown (in open loop) in Palacin et al. (2009a). In this paper new tasks have been incorporated to the operational strategy. In particular a full Model Predictive Control (MPC) has been implemented and a fault detection strategy for correction of fouling in the RO elements has been included.

The problem is formulated in the framework of MPC, as it gives a well-known control approach to incorporate prediction models. Basically, MPC calculates the inputs of the system by an optimization based on the estimated evolution of the system. In order to do that, a dynamic model of the plant is used. In Palacin et al. (2008) a dynamic modeling library of RO plants was presented, that was upgraded and improved in Palacin et al. (2009c). A deeper description of the model can be seen in Syafiie et al. (2008). Some interesting applications of MPC in desalination plants appear in Alatiqi et al. (1999), Gambier et al. (2007b), Bacelli et al. (2009) and Zafra-Cabeza et al. (2009), while in Gambier et al. (2004) the lack of dynamic models for RO plants is commented.

This paper is organized as follows: Section 2 presents a brief description of a typical reverse osmosis plant and the production of drinkable water. Section 3 shows the control problem and the new tasks implemented. Finally, results are shown in section 4 followed by Conclusions and References.

\section{REVERSE OSMOSIS PLANTS}

A typical reverse osmosis plant is shown in Fig. 2. First, a pump (B1) pumps brackish water from a well to a supply tank (T1). From this tank, water is pumped to a set of filters and chemical additions. The high pressure pump (B2) increases its pressure to a value above the osmotic pressure so that the pressurized water can pass then through the RO membrane rack. The difference in pressure between each side of the membranes produces a flow of clean water through the membranes. Finally, this clean water is stored in other tank (T2), which supplies water to the consumers.

Reverse osmosis is a separation process that uses high pressure to force a solvent (water) through a semi permeable membrane that retains the solute (salt) on one side. The clean water flow is called "permeate" and needs a reminarelization before consumed. And the rejected water flow is called "retentate". Typical values of permeate flow are $45 \%$ of the inlet flow for sea water, and $75 \%$ for brackish water. An indepth description of the components of an RO plant could be consulted in Al-Bastaki et al. (1999).

A central problem during operation is the decrease in performance of the membranes, due to deposits (silt, scale, organic components, etc). Thus, in order to prevent precipitation and eliminate microorganisms, a pre-treatment is needed. It normally consists of filtration and the addition of chemical products. In addition, periodic cleanings are scheduled to reduce the amount of deposits. The final part of the process is a post-treatment, to make the water drinkable, by the addition of chlorine and a remineralization.

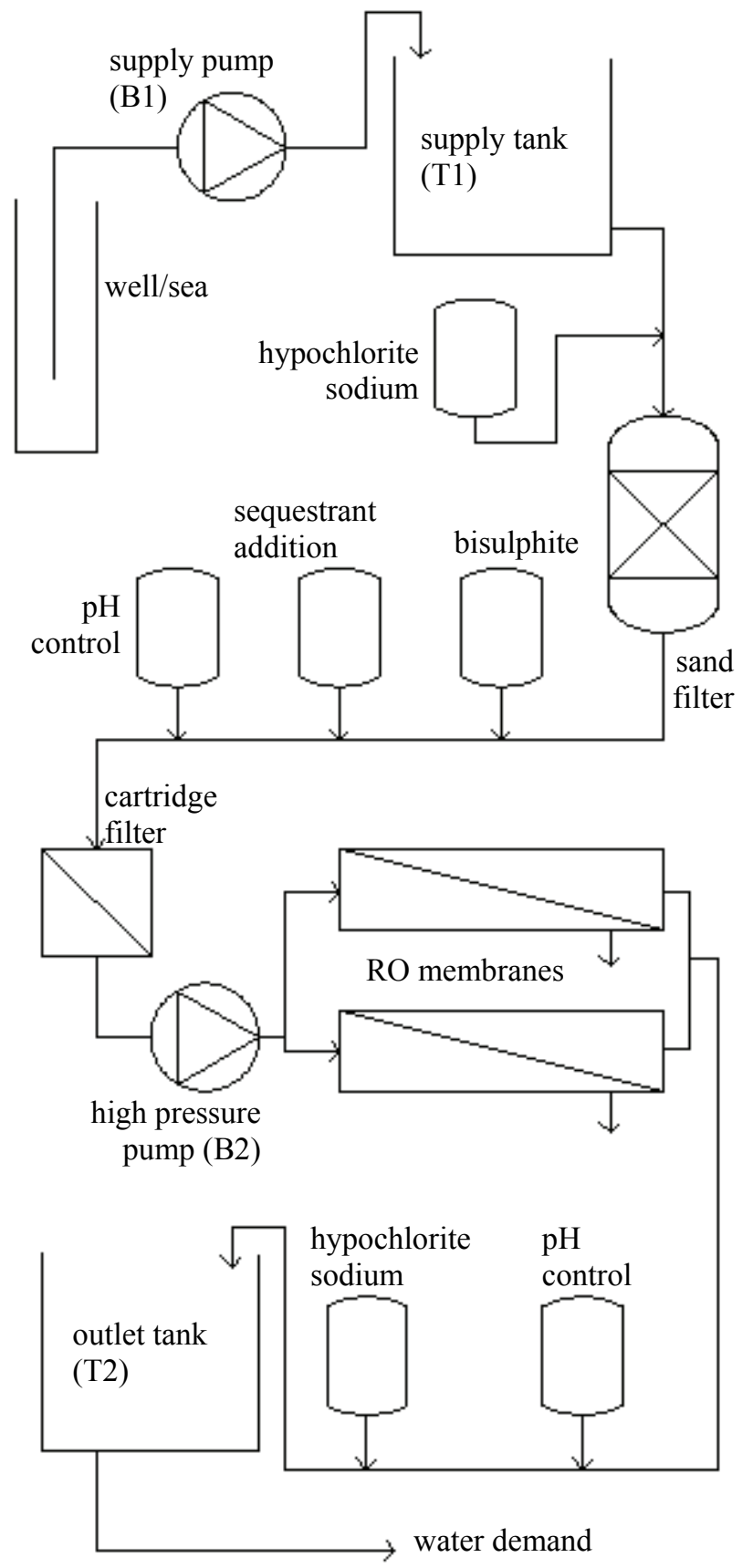

Fig. 2: Typical reverse osmosis plant.

The RO process requires a high pressure on the feed side of the membrane: up to 80 bar for very salty seawater. This pressure is generated by the high pressure pump (B2), that consumes most of the electrical energy needed by the plant. As it has been mentioned, the problem at hand deals with RO plants in which the main sources of energy are renewable energies (solar, wind, etc.), that are strongly variable during a day, so an estimation of its evolution is needed. Backup systems, to provide auxiliary energy, consisting of a diesel generator and batteries are usually needed for the correct operation of RO plants in remote areas: these involve extra cost and their use must be minimized. 


\section{THE CONTROL PROBLEM}

The main objective of the control problem is to fulfill the water demand during a given time horizon, taking into account several constraints. The water demand, which varies during a day, is roughly a periodic curve that is repeated every 24 hours, Tadeo et al. (2009). The forecasting of the characteristic water demand curve is fundamental in a good design of a desalination water plant. This is normally carried out from historical measurements of water demand.

The control objective is formulated jointly with an economic aim: to fulfill the potable water demand while minimizing the energy consumed and using smooth control signals. Notice that, as renewable energies are used, the most important aspect is the minimization of the auxiliary energy consumption. Energy consumption comes from the operation of the pumps of the plant and the cleaning of the membranes. As has been mentioned, the available renewable energy (solar panels and wind generators) can change in the course of a day, so, part of the energy needed is provided from (auxiliary) non renewable energy sources (diesel generator). The control strategy must take into account that auxiliary energy is needed only when the energy consumption of the plant is greater than the available renewable energy. For optimization of the energy consumption, renewable energy predictions are required. There are good models available for solar energy prediction, Alvisi et at. (2007), but short-term predictions of wind energy productions are more difficult, Giebel (2003).

Moreover, membranes and filters cleaning must be performed during certain time periods in order to preserve the membrane life. Notice that when a cleaning operation takes places, no clean water is sent to the storage tank.

In order to fulfill the water demand, the manipulated variables that can be modified in the control problem are:

- $\quad$ flow through the supply pump (Q1).

- flow through the high pressure pump (Q2).

- time instants when membrane cleanings start.

Fig. 3 shows the control structure proposed in this paper, which represents a typical situation. Here, the supply pump (B1) is an on/off centrifugal pump, which can pump a water flow several times higher than the maximum water flow of the high pressure pump (B2). By the contrary, high pressure pump (B2) is a variable speed positive displacement pump able to provide a variable flow.

As the operation of the RO plants mixes continuous decisions (on the flow of water) with discrete ones, such as the cleaning operations or the state of the loading pump, the resulting problem is hybrid in nature. So, initially, a mixed-integer nonlinear programming (MINLP) must be solved. An overview of this type of optimization can be seen in Floudas (1998). However, in order to avoid the use of MINLP, an approach presented in Sarabia et al. (2009) that considers as decision variables the times when the on/off actions take place, instead of binary variables in each sampling time, was used in the MPC framework. These variables are continuous, while the switches can be embedded in the internal model, and, in this way, the problem can be transformed in a non linear programming (NLP) one, which can be easily solved.

So, in order to formulate the optimization problem in practical terms, a vector input parameterization has been used, based on a continuous parameterization, including the time instants of the on/off elements.

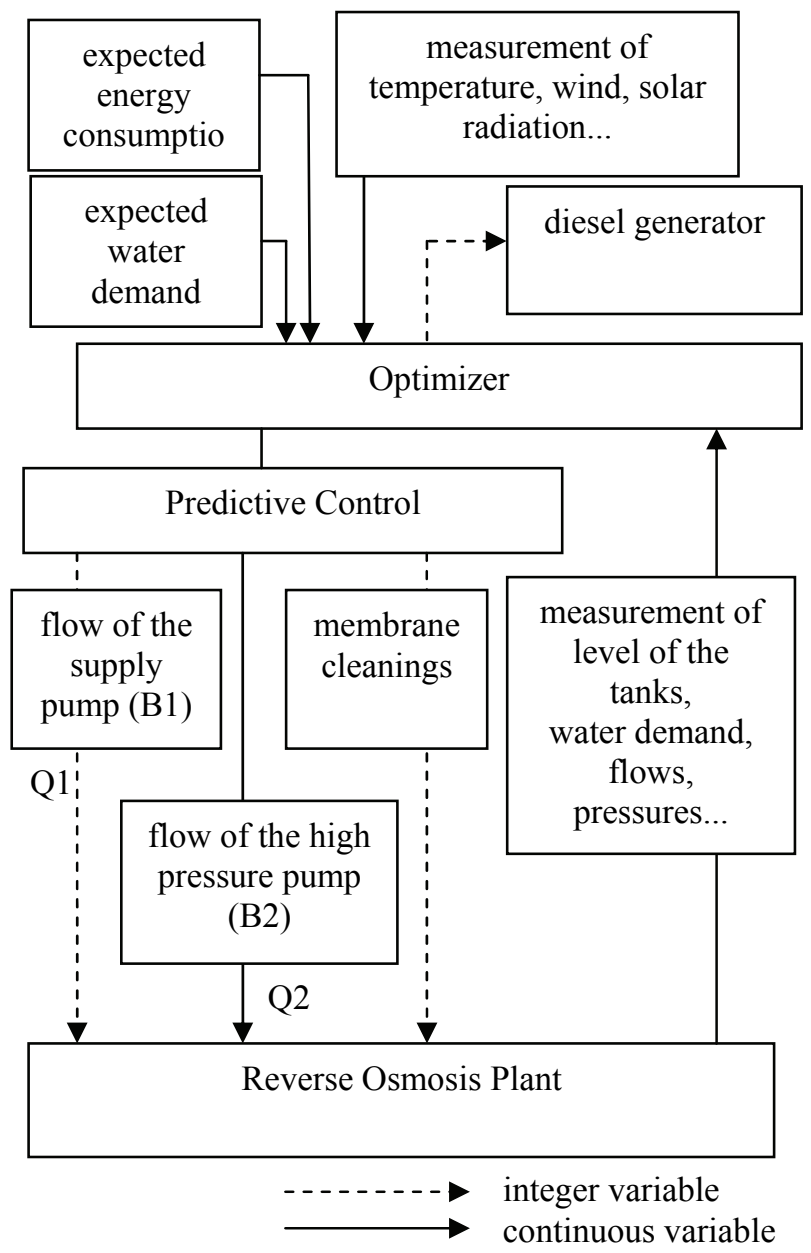

Fig. 3: MPC of the RO plant structure.

Following the previous discussions, there are several constraints on the control problem that have to be taken into account. The main one is keeping the water level of both tanks ( $\mathrm{T} 1$ and $\mathrm{T} 2$ ) between a certain minimum and maximum levels. If the water level of the outlet tank (T2) is kept over a certain minimum, the water demand will be fulfilled. Moreover, several constraints are attached to the manipulated variables:

1. After a switch on of the supply pump (B1), the following switch off cannot be done until a minimum period. In the same way, there is a minimum time between a switch off and the next switch on.

2. In the high pressure pump (B2), the difference between two consecutive changes of pump speed is limited by a maximum value in order to get a smooth operation of the membranes. 
3. As it has been mentioned before, the time between two membrane cleanings should not be higher than 24 hours.

Mathematically, the optimization problem can be formulated as follows:

$\min \beta_{1} \int_{0}^{24 \mathrm{~h}} \mathrm{E}_{\text {auxiliary }} \mathrm{d} \tau+\frac{\beta_{2}}{\mathrm{n}} \sum^{\mathrm{n}} \Delta \mathrm{u}_{\mathrm{B} 2}^{2}$

where $\beta_{l}$ and $\beta_{2}$ are weighting factors. $\mathrm{E}_{\text {auxiliary }}$ is the extra energy consumption, when the renewable energy is insufficient to fulfill the energy consumption (pumps, cleanings, etc.) (kW). $n$ is the number of calculated values of the flow of the high pressure pump (B2) during the control horizon (in this case, the control horizon is 24 hours and $n$ takes the value of 24). $\Delta u_{B 2}$ are the $n$ variations of the speed of the high pressure pump (B2).

The optimization is performed with respect to the vector of decision variables., under the constraints imposed by the model, the admissible range of variables and the constraints 1 to 3 . The dynamic optimization problem is solved using a sequential approach, with the model simulated in the EcosimPro environment, coupled with a SQP optimization routine. Notice that the computation of the gradients can be performed safely, provided that the number of discontinuities does not change over the prediction horizon during its computation, Galan et al. (1999), which is imposed by fixing a number of discrete changes over the horizon.

The optimization is repeated each sample time, and the differences between estimated variables and measurement values are incorporated as corrections as it is usual in MPC. Notice that the hybrid controller will use the water demand predictions, as well as estimations of the available renewable energy, supplied by an energy management system in charge of the wind turbines, solar panels, diesel generators and batteries of the energy subsystem. How to generate these predictions is beyond the scope and length of this paper.

In addition, a common fault treatment has been included in the formulation. It corresponds to calcium carbonate fouling. A deep explanation of this problem can be seen in Elfil et al. (2004) and Elfil et al. (2006). This fouling can be avoided by a correct $\mathrm{pH}$ control of the feed flow of the membrane. It can be detected by the measurement of the concentrate flow during cleanings. Fig. 4 shows typical $\mathrm{pH}$ curves for normal cleaning and for operation with calcium carbonate fouling. When a carbonate fouling is detected through the $\mathrm{pH}$ measurement, different measures can be taken. In general, an extra cleaning of the membrane with a given delay can be used. A study of fault detection techniques can be seen in Venkatasubramanian et al. (2003). A detection mechanism is incorporated and, when activated, the control policy is changed incorporating the double cleaning.

\section{SIMULATION RESULTS}

This section shows the predictive control of the RO plant during two days. The control and prediction horizon of the MPC are 24 hours, while the time step for the controller is 1 hour. Results of the closed loop operation are given in the figures 5-10. Fig. 5 displays the real and the estimated water demand flows. Differences between both curves were included in order to test the controller in more realistic conditions. Fig. 6 shows the input and output flows of the supply tank, T1. Input flow of this tank is the flow pumped by B1, and output flow is the feed flow of the membranes. Next, input and output flows of the outlet tank, T2, are shown in Fig. 7. Input flow of this second tank is equal to the permeate flow of the membranes (manipulated variable), and output flow is the water demand. Membrane cleanings are shown in figures 6 and 7 and corresponds to the time instants where feed flow and permeate flow are zero.

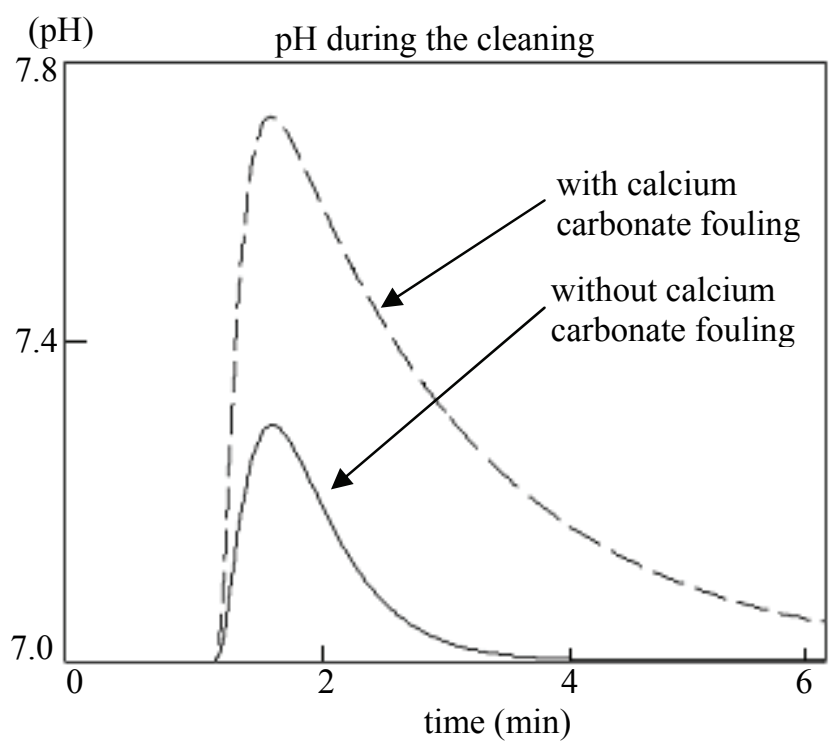

Fig. 4: Membrane fouling detection by the $\mathrm{pH}$ measurement during the cleaning.

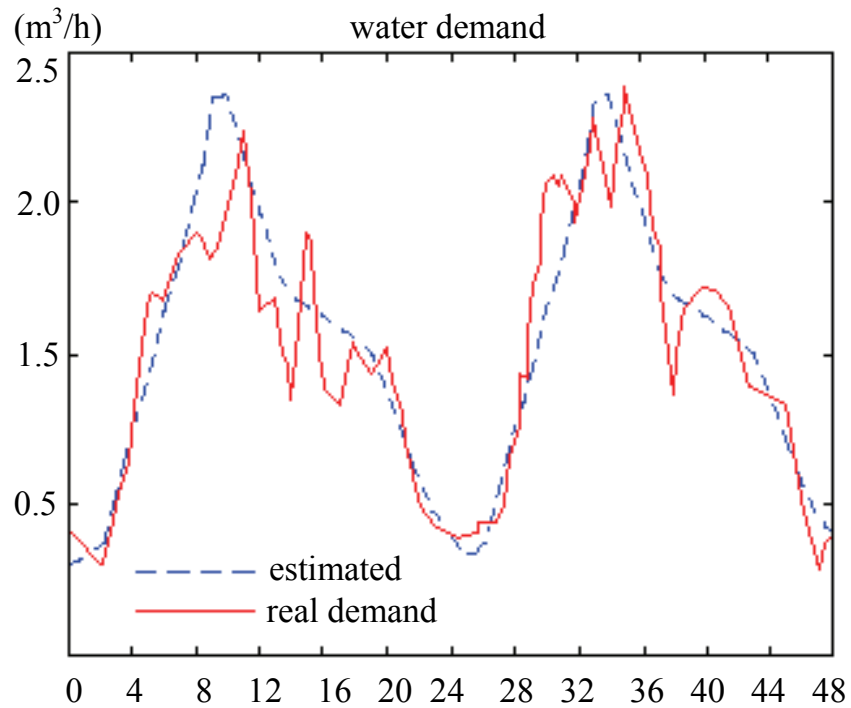

Fig. 5: Water demand (estimated curve and real demand) during 48 hours.

Fig 8 shows the water level in both tanks. Notice that both levels are kept between two given minimum and maximum levels, thus guaranteeing the water supply at all times. 


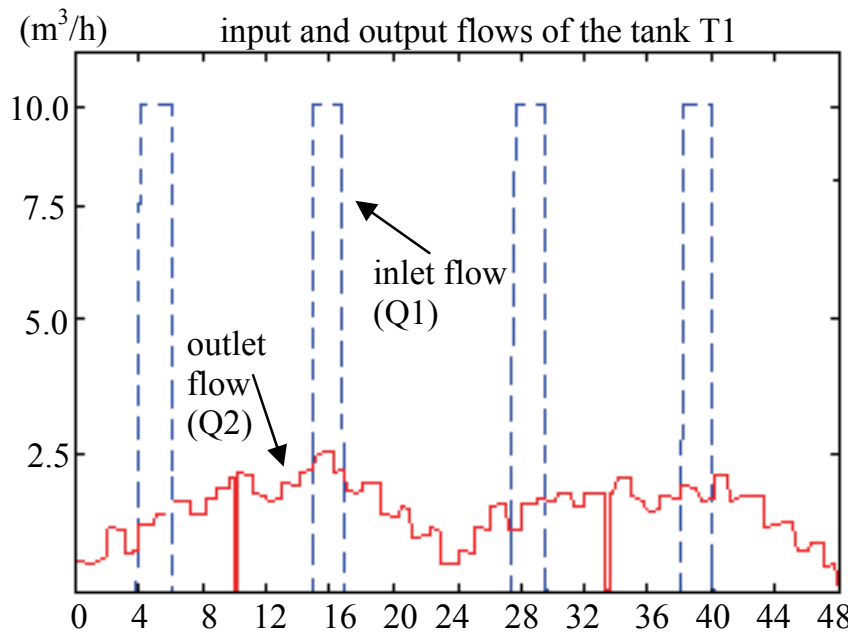

Fig. 6: The manipulated variables, the input flow (Q1) and output flow (Q2) of the tank T1, during 48 hours.

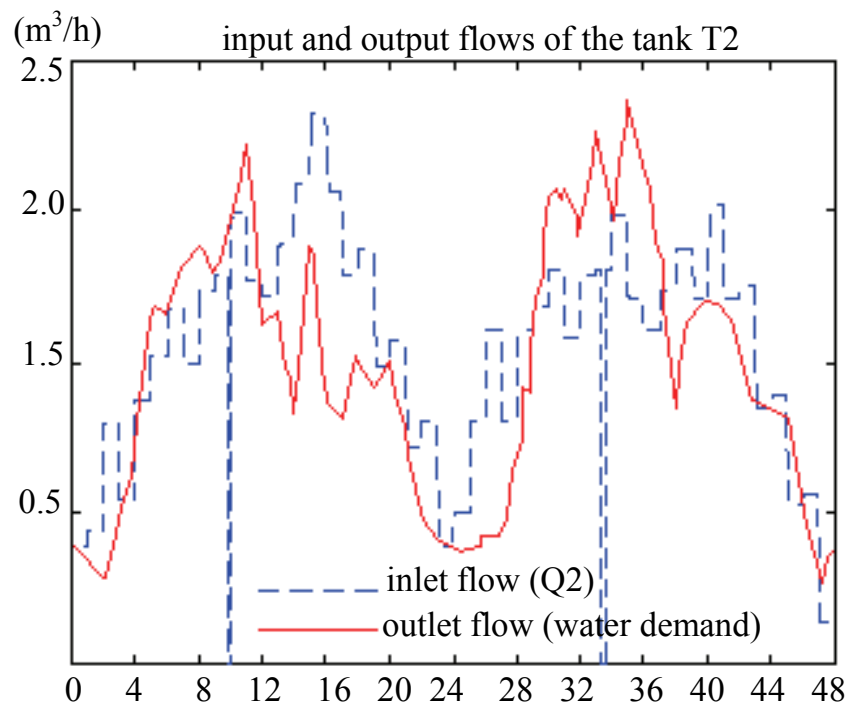

Fig. 7: Input and output flow of the tank T2, during 48 hours. The manipulated variables are the output flow, Q2, and the time instants when membrane cleanings start (at time $=10$ and time $=33$ hours).

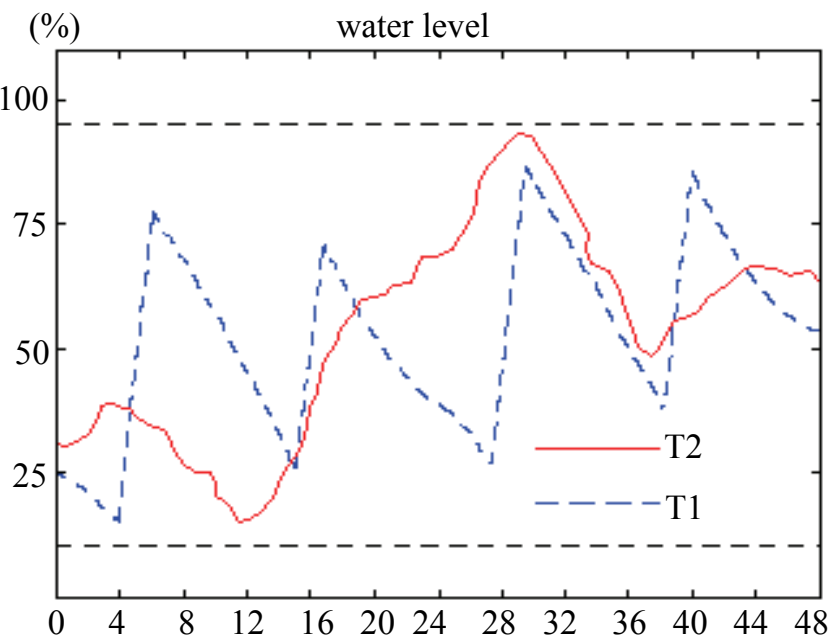

Fig. 8: Water level of both tanks during 48 hours.

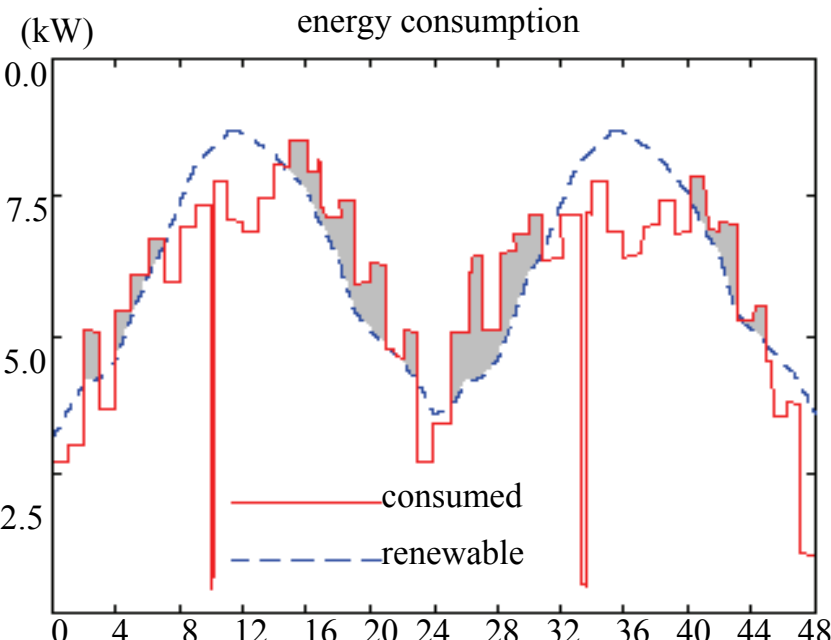

Fig. 9: Energy consumption by the RO plant and available renewable energy, during 48 hours. Notice the membrane cleanings at time $=10$ and 33 hours.

Fig. 9 displays the energy consumption of the RO plant and the available renewable energy. Besides, the auxiliary energy consumption is displayed as grey areas. The auxiliary energy is needed when the renewable energy is insufficient to fulfill the energy consumption of the RO plant.

Finally, a simulation of the fouling by calcium carbonate is shown in Fig. 10 with the double cleaning, frequently used to eliminate the deposits.

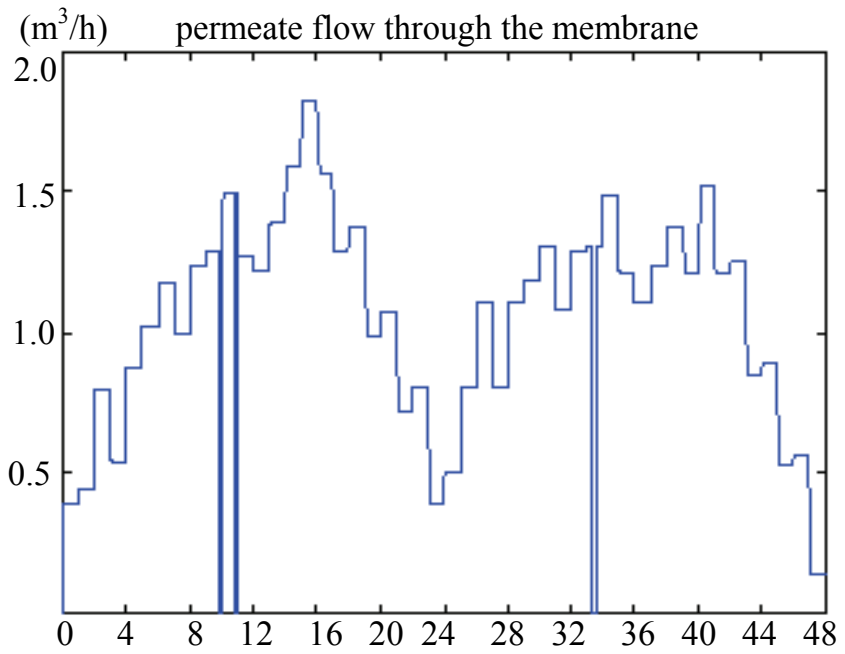

Fig. 10: Permeate flow, during 48 hours. Notice than two cleanings have been done at time 10 and 10.5 hours, in order to eliminate the calcium carbonate fouling.

\section{CONCLUSIONS}

This paper presents a hybrid control algorithm for the optimal operation of medium-size reverse osmosis plants, coupled with autonomous energy sources. The proposed approach is devised to improve efficiency, extend the life of the components and reduce installation and operation costs. The predictive controller uses hourly predictions of water demand to adequately schedule the cleaning times and the operation of the pumps, taking into account the different restrictions 
imposed by components of the plant. Preliminary results are promising, in the sense that good control of the system is achieved, fulfilling the control objectives without excessive use of auxiliary energy. Finally, a simulation with calcium carbonate fouling has been shown, to see how this fouling can be detected and corrected.

\section{ACKNOWLEDGMENT}

The authors wish to express their gratitude to the Spanish MICINN for its support through project DPI2006-13593, as well as the EU for its support through 6FP, OPEN-GAIN contract 032535

\section{REFERENCES}

Alatiqi, I., Ettouney, H., and El-Dessouky, H. (1999). Process control in water desalination industry: an overview. Desalination, (126), 15-32.

Al-Bastaki, N. M., and Abbas, A. (1999) Modeling an industrial reverse osmosis unit. Desalination, (126), 3339.

Alvisi, S., Franchini, M., and Marinelli. A. (2007). A shortterm, pattern-based model for water-demand forecasting. Journal of Hydroinformatics. (9), 39-50.

Bacelli, G., Gilloteaux, J.C., and Ringwood, J. (2009). A predictive controller for a heaving buoy producing producing potable water. Proc of the European control conference 2009. Budapest, Hungary.

Baker, R. (2004). Membrane technology and applications. Wiley, West Sussex, England, UK.

Elfil, H., and Roques, H. (2004). Prediction of the limit of the metastable zone in the "CaCO3-CO2-H2O" system. AIChe Journal. (50), 1908-1916.

Elfil, H., and Roques, H. (2006). Reconsidering water scaling tendency assessment. AIChe Journal. (52), 3583-3590.

Floudas, C.A., (1998) Nonlinear and mixed-integer optimization. fundamentals and applications. Journal of global optimization. Springer Netherlands. (12), 108-110.

Fritzmann, C., Löwenberg, J., Wintgens, T., and Melin, T. (2007). State-of-the-art of reverse osmosis desalination. Desalination, (216), 1-76.

Galan, S., Feehery, W.F. and Barton, P.I. (1999). Parametric sensitivity functions for hybrid discrete/continuous systems. Applied Numerical Mathematics, (31), 17.47.

Gambier, A. and Badreddin. E. (2004), Dynamic modelling of MSF plants for automatic control and simulation purposes: a survey. Desalination, (166), 191-204.

Gambier, A., Krasnik, A., and Badreddin, E. (2007). Modeling of a simple reverse osmosis desalination plant for advanced control purposes. Proc of the American control conference. New York, USA.

Gambier, A., Krasnik, A., and Badreddin, E. (2007). Dynamic modeling of a simple reverse osmosis desalination plant for advanced control purposes, Proc. of the American control conference. New York, USA, 2007.

Giebel, G. (2003). The state-of-the-art in shor-term prediction of wind power. Project ANEMOS ENK5-CT2002-00665

Palacin, L., de Prada, C., Syafiie, S., and Tadeo, F. (2008). Library for dynamic simulation of reverse osmosis plants. Proc. of 20th European modeling and simulation symposium. Briatico, Italy.

Palacin, L., Tadeo, F., and de Prada, C. (2009). Operation of desalination plants using hybrid control. Proc. of the 2 nd Maghreb conference on desalination and water treatment. Hammamet, Tunisia.

Palacin, L., Tadeo, F., and de Prada, C. (2009). Integrated design using dynamic simulation of reverse osmosis plants. Proc. of the international conference on industrial engineering and engineering management, IEEE 2009. Hong Kong, China.

Palacin, L., Tadeo, F., Elfil, H., and de Prada, C. (2009). New dynamic library of reverse osmosis plants with fault simulation. Proc. of the 2nd Maghreb conference on desalination and water treatment. Hammamet, Tunisia.

Porter, M.C. (1990). Handbook of industrial membrane technology. Noyes publications, Westwood, New Jersey, USA.

Salazar, J., Tadeo, F., and de Prada, C. (2010). Control system for reverse osmosis plant (RO) and renewable energy generation. Proc of the international conference on renewable energies and power quality 2010, Granada, Spain

Sarabia, D., Capraro, F., Larsen, L.F.S., and de Prada, C. (2009). Hybrid NMPC of supermarket display cases, Control Engineering Practice. (17) 428-441

Syafiie, S., Palacin, L., de Prada, C., and Tadeo, F. (2008). Membrane modeling for simulation and control of reverse osmosis in desalination plants. Proc. of control 2008. Manchester, UK.

Tadeo, F. Val, R., Palacin, L., Salazar, J., and de Prada, C. (2009). Control of reverse osmosis plants using renewable energies. Proc. of control and applications 2009, Cambridge, UK.

Venkatasubramanian, V., Rengaswamy, R., and Kavuri, S.N. (2003). A review of process fault detection and diagnosis. Computers and Chemical Engineering. (27), 327-346.

Wilf, M. (2007). The guidebook to membrane desalination technology. Balaban desalination publication, L'Aquila, Italy.

Zafra-Cabeza, A., Ridao, M.A., and Camacho, E.F. (2009). Optimization in reverse osmosis plants through risk mitigation. Proc of the European control conference 2009. Budapest, Hungary. 